PROCEEDINGS OF THE

AMERICAN MATHEMATICAL SOCIETY

Volume 133, Number 4, Pages 979-985

S 0002-9939(04)07638-5

Article electronically published on September 16, 2004

\title{
DETECTING THE INDEX OF A SUBGROUP IN THE SUBGROUP LATTICE
}

\author{
M. DE FALCO, F. DE GIOVANNI, C. MUSELLA, AND R. SCHMIDT
}

(Communicated by Jonathan I. Hall)

\begin{abstract}
A theorem by Zacher and Rips states that the finiteness of the index of a subgroup can be described in terms of purely lattice-theoretic concepts. On the other hand, it is clear that if $G$ is a group and $H$ is a subgroup of finite index of $G$, the index $|G: H|$ cannot be recognized in the lattice $\mathfrak{L}(G)$ of all subgroups of $G$, as for instance all groups of prime order have isomorphic subgroup lattices. The aim of this paper is to give a lattice-theoretic characterization of the number of prime factors (with multiplicity) of $|G: H|$.
\end{abstract}

\section{INTRODUCTION}

For every group $G$, we shall denote by $\mathfrak{L}(G)$ the lattice of all subgroups of $G$. If $G$ and $\bar{G}$ are groups, an isomorphism from the lattice $\mathfrak{L}(G)$ onto the lattice $\mathfrak{L}(\bar{G})$ is also called a projectivity from $G$ onto $\bar{G}$; one of the main problems in the theory of subgroup lattices is to find group properties that are invariant under projectivities. In 1980, Zacher [5] and Rips proved independently that any projectivity from a group $G$ onto a group $\bar{G}$ maps each subgroup of finite index of $G$ to a subgroup of finite index of $\bar{G}$. In addition, Zacher gave a lattice-theoretic characterization of the finiteness of the index of a subgroup in a group; other characterizations were given by Schmidt [3. On the other hand, it is clear that if $G$ is a group and $H$ is a subgroup of finite index of $G$, the index $|G: H|$ cannot be recognized in the subgroup lattice $\mathfrak{L}(G)$, as for instance all groups of prime order have the same lattice of subgroups.

The aim of this paper is to find an arithmetic invariant related to the index of a subgroup and preserved under projectivities. In fact, if $H$ is a subgroup of finite index of any group $G$, we will give a lattice-theoretic characterization of the number of prime factors (with multiplicity) of $|G: H|$, so that this number can be detected in the lattice $\mathfrak{L}(G)$.

Most of our notation is standard and can be found in [2]; for definitions and properties concerning lattices and subgroup lattices we refer to the monograph [4. In particular, if $\mathfrak{L}$ is any complete lattice, the smallest and the largest element of $\mathfrak{L}$ will be denoted by 0 and $I$, respectively; moreover, for each pair $(a, b)$ of elements of $\mathfrak{L}$ such that $a \leq b$, we put $[b / a]=\{x \in \mathfrak{L} \mid a \leq x \leq b\}$. If $a$ is any non-zero

Received by the editors October 8, 2003 and, in revised form, December 1, 2003.

2000 Mathematics Subject Classification. Primary 20E15.

(C)2004 American Mathematical Society Reverts to public domain 28 years from publication 
element of the finite lattice $\mathfrak{L}$, we put

$$
\phi(a)=\inf \{x \in \mathfrak{L} \mid x<\cdot a\}
$$

(where the symbol $x<\cdot a$ means that $x$ is a maximal (proper) element of the lattice $[a / 0])$. Finally, for each positive integer $n$ we will denote by $M_{n}$ the lattice of length 2 with $n$ atoms.

\section{The Weight of A Finite LAtTice}

A finite lattice $\mathfrak{L}$ is called perfect if it has no maximal elements that are modular.

Lemma 2.1. Let $\mathfrak{L}$ be a finite lattice, and let $x$ and $y$ be elements of $\mathfrak{L}$ such that the intervals $[x / 0]$ and $[y / 0]$ are perfect lattices. Then also the lattice $[x \vee y / 0]$ is perfect.

Proof. Assume for a contradiction that $[x \vee y / 0]$ contains a maximal element $z$ that is modular. Since $x \wedge z$ is modular in the perfect lattice $[x / 0]$ and the lattices $[x \vee z / z]$ and $[x / x \wedge z]$ are isomorphic, it follows that $x \wedge z=x$ and hence $x \leq z$. We obtain similarly that $y \leq z$ and so $z=x \vee y$, a contradiction. Therefore the lattice $[x \vee y / 0]$ is perfect.

Let $\mathfrak{L}$ be a finite lattice. It follows from Lemma 2.1 that $\mathfrak{L}$ contains a largest element $r$ such that the interval $[r / 0]$ is a perfect lattice; such an element $r$ will be called the perfect radical of $\mathfrak{L}$ and denoted by $r(\mathfrak{L})$. Clearly, the lattice $\mathfrak{L}$ is perfect if and only if $r(\mathfrak{L})=I$.

Recall that an element $c$ of a finite lattice $\mathfrak{L}$ is called cyclic if the interval $[c / 0]$ is a distributive lattice. Moreover, an element $a$ of $\mathfrak{L}$ is said to be modularly embedded in $\mathfrak{L}$ if the interval $[a \vee c / 0]$ is a modular lattice for each cyclic element $c$ of $\mathfrak{L}$; a modular chain in $\mathfrak{L}$ is a chain of elements of $\mathfrak{L}$ of the form

$$
0=a_{0}<a_{1}<\ldots<a_{t}=I
$$

such that $a_{i+1}$ is modularly embedded in $\left[I / a_{i}\right]$ for each non-negative integer $i<t$.

For our purposes, we will consider the subset $P(\mathfrak{L})$ of $\mathfrak{L}$ consisting of all elements $a$ satisfying the following conditions:

- the lattice $[a / 0]$ has a modular chain;

- every interval of $[a / 0]$ is directly indecomposable;

- if $x<\cdot y \leq a$ and $[x / 0]$ is a chain of length 2 , then either $[y / 0]$ is a modular lattice or it is isomorphic to the subgroup lattice $\mathfrak{L}\left(D_{8}\right)$ of the dihedral group of order 8.

In particular, $P(\mathfrak{L})$ contains any element $a$ of $\mathfrak{L}$ such that $[a / 0]$ is a modular lattice whose intervals are directly indecomposable. Note also that, in the special case of the subgroup lattice of a finite group $G$, it turns out that the elements of $P(\mathfrak{L}(G))$ are precisely the primary subgroups and the $P$-subgroups of $G$ (see [4], Theorem 7.4.10). Here a group is called a $P$-group if it is the semidirect product of an abelian normal subgroup $A$ of prime exponent by a group $\langle x\rangle$ of prime order such that $x$ induces on $A$ a power automorphism; in particular, all abelian groups of prime exponent are $P$-groups.

For a finite lattice $\mathfrak{L}$, we let $A_{\mathfrak{L}}$ be the set of all atoms of $\mathfrak{L}$. For every prime number $p$, we define two subsets of $A_{\mathfrak{L}}$, namely

$$
R_{\mathfrak{L}}(p)=\left\{a \in A_{\mathfrak{L}} \mid \exists b \in P(\mathfrak{L}) \text { such that } a \leq b \text { and }[b / \phi(b)] \simeq M_{p+1}\right\}
$$


and

$$
\begin{aligned}
S_{\mathfrak{L}}(p)= & \left\{a \in A_{\mathfrak{L}} \mid \exists b \in \mathfrak{L} \text { such that }[b / 0] \text { is a chain, } \phi(b) \neq 0,\right. \\
& {\left.[a \vee \phi(b) / 0] \text { is distributive and }[a \vee b / \phi(b)] \simeq M_{p+1}\right\} ; }
\end{aligned}
$$

furthermore, we let $T_{\mathfrak{L}}(p)=R_{\mathfrak{L}}(p) \cup S_{\mathfrak{L}}(p)$ and

$$
T_{\mathfrak{L}}=\bigcup_{p \in \mathbb{P}} T_{\mathfrak{L}}(p)
$$

where $\mathbb{P}$ is the set of all prime numbers. Then, clearly, $T_{\mathfrak{L}} \subseteq A_{\mathfrak{L}}$ and in general $T_{\mathfrak{L}} \neq A_{\mathfrak{L}}$, for instance if $\mathfrak{L}$ is a non-trivial chain. Finally, for every atom $a$ of $\mathfrak{L}$, we define

$$
\omega_{\mathfrak{L}}(a)=\operatorname{Min}\left\{p \in \mathbb{P} \mid a \in T_{\mathfrak{L}}(p)\right\}
$$

if $a \in T_{\mathfrak{L}}$ and $\omega_{\mathfrak{L}}(a)=0$ if $a \in A_{\mathfrak{L}} \backslash T_{\mathfrak{L}}$. Then

$$
\omega_{\mathfrak{L}}: A_{\mathfrak{L}} \longrightarrow \mathbb{P} \cup\{0\}
$$

is a well-defined map described entirely in the (finite) lattice $\mathfrak{L}$.

An element $x \in \mathfrak{L}$ is called a p-element of $\mathfrak{L}$ if $\omega_{\mathfrak{L}}(a)=p$ for every atom $a$ of $[x / 0]$. As usual, the length $l(\mathfrak{L})$ of $\mathfrak{L}$ is the largest length of a chain in $\mathfrak{L}$, and we denote the largest length of a chain consisting of $p$-elements in $\mathfrak{L}$ by $\ell_{p}(\mathfrak{L})$. The weight $\|\mathfrak{L}\|$ of $\mathfrak{L}$ is now defined by

$$
\|\mathfrak{L}\|=\ell([I / r(\mathfrak{L})])+\sum_{p \in \mathbb{P}} \ell_{p}([r(\mathfrak{L}) / 0]),
$$

where $r(\mathfrak{L})$ is the perfect radical of $\mathfrak{L}$ defined above.

\section{THE ORDER OF A FINITE GROUP}

It is well known that a finite group is perfect if and only if its subgroup lattice is perfect (see [4], Theorem 5.3.3). It follows that for any finite group $G$, the perfect

radical of the lattice $\mathfrak{L}(G)$ is the largest perfect subgroup of $G$ (and so it coincides with the soluble residual of $G$ ).

Lemma 3.1. Let $H$ be a minimal subgroup of a finite group $G$, and let $p$ be a prime number. If $H \in S_{\mathfrak{L}(G)}(p)$, then $|H|=p$.

Proof. Since $H \in S_{\mathfrak{L}(G)}(p)$, there exists a cyclic subgroup $K$ of prime power order such that $\phi(K) \neq\{1\},\langle H, \phi(K)\rangle$ is cyclic and

$$
[\langle H, K\rangle / \phi(K)] \simeq M_{p+1} .
$$

Thus $H$ is not contained in $K$, so that $\langle H, \phi(K)\rangle=H \times \phi(K)$, and in particular $H$ and $K$ have coprime orders. So $\langle H, K\rangle / \phi(K)$ cannot be a $p$-group, and hence it is non-abelian of order $p q$ where $p>q \in \mathbb{P}$. Thus $[H, K] \neq\{1\}$ and $[H, \phi(K)]=\{1\}$. Therefore $K$ cannot be normal in $\langle H, K\rangle$, and it follows that $|K / \phi(K)|=q$ and $|H|=p$.

Lemma 3.2. Let $G$ be a finite group having no normal Sylow complement. Then $\omega_{\mathfrak{L}(G)}(H)=|H|$ for every minimal subgroup $H$ of $G$. 
Proof. Let $|H|=p$. We claim that it suffices to show that $H$ belongs to $T_{\mathfrak{L}(G)}(p)$. Indeed, this clearly implies that $0<\omega_{\mathfrak{L}(G)}(H) \leq p$. If $H$ were contained in $T_{\mathfrak{L}(G)}(q)$ for some prime $q<p$, then by Lemma 3.1, $H \in R_{\mathfrak{L}(G)}(q)$ and so there would exist $Q \in P(\mathfrak{L}(G))$ such that $H \leq Q$ and $[Q / \phi(Q)] \simeq M_{q+1}$. In this case, $Q$ would be a $q$-group or a $P$-group of order $q r$ with $q \geq r \in \mathbb{P}$ (see 4], Theorem 7.4.10). This would contradict the fact that $H \leq Q$ and $|H|=p>q$. Thus $\omega_{\mathfrak{L}(G)}(H)=p=|H|$.

To prove that $H \in T_{\mathfrak{L}(G)}(p)$, consider a Sylow $p$-subgroup $S$ of $G$ containing $H$. If $S$ is not cyclic, then we may consider a smallest non-cyclic subgroup $P$ of $S$ containing $H$. Every maximal subgroup of $P$ containing $H$ is cyclic and hence $[P / \phi(P)] \simeq M_{p+1}$; thus $H \in R_{\mathfrak{L}(G)}(p)$. So suppose that $S$ is cyclic. Since $G$ is not $p$-nilpotent, we have $S \leq C_{G}(S)<N_{G}(S)$ (see [2, 10.1.8), and for some prime $q \neq p$ there exists an element $g \in N_{G}(S)$ with order $q^{n}$ inducing an automorphism of order $q$ in $S$. Then $\phi(\langle g\rangle)=\left\langle g^{q}\right\rangle$ centralizes $S$, and in particular the subgroup $\langle H, \phi(\langle g\rangle)\rangle$ is cyclic; furthermore, $\langle H, g\rangle / \phi(\langle g\rangle)$ is non-abelian of order $p q$ and hence $[\langle H, g\rangle / \phi(\langle g\rangle)] \simeq M_{p+1}$. So if $\phi(\langle g\rangle) \neq\{1\}$, then $H \in S_{\mathfrak{L}(G)}(p)$; and if $\phi(\langle g\rangle)=\{1\}$, then $\langle H, g\rangle \in P(\mathfrak{L}(G))$ and $H \in R_{\mathfrak{L}(G)}(p)$. In all cases, $H \in T_{\mathfrak{L}(G)}(p)$ as we wanted to show.

We can now prove the following result, which provides a purely lattice-theoretic description of the order of a finite group having no normal Sylow complement, in particular of any finite perfect group.

Theorem 3.3. Let $G$ be a finite group having no normal Sylow complement. Then $|G|=\prod_{p \in \mathbb{P}} p^{\ell_{p}(\mathfrak{L}(G))}$

Proof. It follows from Lemma 3.2 that for each prime number $p$, the $p$-elements of the lattice $\mathfrak{L}(G)$ are precisely the $p$-subgroups of $G$. In particular, if $P$ is any Sylow $p$-subgroup of $G$, we have that $|P|=p^{\ell_{p}(\mathfrak{L}(G))}$. The theorem follows.

For an arbitrary finite group $G$, the order of $G$ cannot be recognized in $\mathfrak{L}(G)$. But we can describe the number of prime factors of $|G|$ in $\mathfrak{L}(G)$.

Theorem 3.4. Let $G$ be a finite group. Then the weight $\|\mathfrak{L}(G)\|$ of the subgroup lattice of $G$ is the number of prime factors of the order of $G$ (with multiplicity).

Proof. Let $R$ be the soluble residual of $G$. Then $R=r(\mathfrak{L}(G))$ and Theorem 3.3 yields that

$$
\sum_{p \in \mathbb{P}} \ell_{p}([r(\mathfrak{L}(G)) / 0])=\sum_{p \in \mathbb{P}} \ell_{p}(\mathfrak{L}(R))
$$

is the number of prime factors of $|R|$. Since $G / R$ is soluble, the number of prime factors of $|G / R|$ is just the length of the lattice $\mathfrak{L}(G / R)$. The number of prime factors of $|G|$ is the sum of these two numbers, and hence it is $\| \mathfrak{L}(G)) \|$.

The above theorem has the following obvious consequence.

Corollary 3.5. Let $H$ be a subgroup of the finite group $G$. Then the number of prime factors of the index $|G: H|$ (with multiplicity) is $\|\mathfrak{L}(G)\|-\|\mathfrak{L}(H)\|$. 


\section{Subgroups of Finite indeX}

It is well known that if $a$ and $b$ are modular elements of a lattice $\mathfrak{L}$, then also $a \vee b$ is a modular element of $\mathfrak{L}$; in the case of the lattice of all subgroups of a group $G$, it has been proved that the join of any collection of modular subgroups of $G$ is likewise a modular subgroup (see [1], Proposizione 1.2). As G. Zacher pointed out to one of the authors, this property also holds for arbitrary algebraic lattices (recall that a complete lattice $\mathfrak{L}$ is called algebraic if each element of $\mathfrak{L}$ is a join of compact elements).

Lemma 4.1. Let $\mathfrak{L}$ be an algebraic lattice, and let $X$ be a non-empty set of modular elements of $\mathfrak{L}$. Then also $\sup X$ is a modular element of $\mathfrak{L}$.

Proof. Put $a=\sup X$, and let $b$ be any element of $\mathfrak{L}$. Consider an element $y$ of the interval $[a \vee b / a]$, and let $\left(y_{i}\right)_{i \in I}$ be a collection of compact elements of $\mathfrak{L}$ such that $y=\sup _{i \in I} y_{i}$. For each $i \in I$ there exists a finite subset $X_{i}$ of $X$ such that $y_{i} \leq x_{i} \vee b$, where $x_{i}=\sup X_{i}$; clearly, $x_{i}$ is a modular element of $\mathfrak{L}$, and hence

$$
y_{i} \leq y \wedge\left(x_{i} \vee b\right)=x_{i} \vee(b \wedge y) \leq a \vee(b \wedge y)
$$

Thus $y \leq a \vee(b \wedge y)$, and so $a \vee(b \wedge y)=y$.

Suppose now that $z$ is an element of the interval $[b / a \wedge b]$, and put $c=(a \vee z) \wedge b$. Let $\left(c_{j}\right)_{j \in J}$ be a collection of compact elements of $\mathfrak{L}$ for which $c=\sup _{j \in J} c_{j}$, and for each $j \in J$ let $X_{j}^{\prime}$ be a finite subset of $X$ such that $c_{j} \leq x_{j}^{\prime} \vee z$, where $x_{j}^{\prime}=\sup X_{j}^{\prime}$. Since $x_{j}^{\prime}$ is a modular element of $\mathfrak{L}$, we have

$$
c_{j} \leq\left(x_{j}^{\prime} \vee z\right) \wedge b=z \vee\left(x_{j}^{\prime} \wedge b\right)=z,
$$

so that $c \leq z$ and hence $z=c=(a \vee z) \wedge b$. It follows that $a$ is a modular element of $\mathfrak{L}$ (see [4, Theorem 2.1.5).

Let $\mathfrak{L}$ be an algebraic lattice, and let $a$ be any element of $\mathfrak{L}$. The largest modular element $m$ of $\mathfrak{L}$ such that $m \leq a$ is called the modular core of $a$ in $\mathfrak{L}$, and is denoted by core $\cos _{\mathfrak{L}} a$. Clearly, the element $a$ is modular if and only if $a=\operatorname{core}_{\mathfrak{L}} a$; note also that if core $_{\mathfrak{L}} a<a$, then $a$ cannot be modular in the lattice $\left[I /\right.$ core $\left._{\mathfrak{L}} a\right]$. If $a$ and $b$ are elements of $\mathfrak{L}$ such that $a<b$, the modular core of $a$ in $[b / 0]$ will also be denoted by core $_{b} a$.

Let $\mathfrak{L}$ be an infinite algebraic lattice. A maximal element $a$ of $\mathfrak{L}$ is called $f$ maximal if the interval $[a / 0]$ is infinite and $a$ satisfies one of the following conditions:

(1) $a$ is not modular in $\mathfrak{L}$ and $\left[I / \operatorname{core}_{\mathfrak{L}} a\right]$ is a finite lattice;

(2) there exists an automorphism $\varphi$ of $\mathfrak{L}$ such that $a \wedge a^{\varphi}$ is a modular element of $\mathfrak{L}$ and $\left[I / a \wedge a^{\varphi}\right]$ is a finite lattice with length 2 and at least 3 atoms;

(3) for each automorphism $\varphi$ of $\mathfrak{L}$, the element $a \wedge a^{\varphi}$ is modular in $\mathfrak{L}$ and $\left[I / a \wedge a^{\varphi}\right]=\left\{a \wedge a^{\varphi}, a, a^{\varphi}, I\right\}$.

It follows from the definition that if $a$ is any $f$-maximal element of $\mathfrak{L}$, the lattice $\left[I / \operatorname{core}_{\mathfrak{L}} a\right]$ is finite; note also that both conditions (2) and (3) above force the element $a$ to be modular in $\mathfrak{L}$.

Let $\mathfrak{L}$ be an infinite algebraic lattice, and let $a$ and $b$ be elements of $\mathfrak{L}$ such that $a<b$ and $a$ is $f$-maximal in $[b / 0]$; since the lattices $\left[a /\right.$ core $\left._{b} a\right]$ and $\left[b /\right.$ core $\left._{b} a\right]$ are finite, we can define the lattice index $\|b: a\|$ of $a$ in $b$ by the position

$$
\|b: a\|=\|\left[b / \text { core }_{b} a\right]\|-\|\left[a / \text { core }_{b} a\right] \| .
$$


In particular, if $a$ is an $f$-maximal and modular element of $[b / 0]$, we have $\|b: a\|=$ $\|[b / a]\|=1$.

Let $G$ be an infinite group; a subgroup $M$ of $G$ is called $f$-maximal if $M$ is an $f$-maximal element of the lattice $\mathfrak{L}(G)$. Actually, the $f$-maximal subgroups of $G$ are precisely the maximal subgroups of finite index; in fact, the following lattice characterization of the finiteness of the index of a subgroup holds.

Lemma 4.2. Let $G$ be an infinite group, and let $H$ be a proper subgroup of $G$. Then $H$ has finite index in $G$ if and only if there exists a finite chain $H=H_{0}<$ $H_{1}<\ldots<H_{t}=G$ such that $H_{i}$ is an $f$-maximal subgroup of $H_{i+1}$ for each $i=0,1, \ldots, t-1$.

Proof. Suppose first that the index $|G: H|$ is finite, and let

$$
H=H_{0}<H_{1}<\ldots<H_{t}=G
$$

be a maximal chain of subgroups between $H$ and $G$. Then the subgroup $H_{i}$ is infinite and maximal in $H_{i+1}$ for each $i=0,1, \ldots, t-1$; moreover, since $\left|H_{i+1}: H_{i}\right|$ is finite, we have that $H_{i}$ is an $f$-maximal subgroup of $H_{i+1}$ (see 3, Satz 3). The converse statement follows from the same result.

We also need the following known result; it shows that if $M$ is an $f$-maximal subgroup of an infinite group $G$, then either $\operatorname{core}_{\mathfrak{L}(G)} M=M$ or $_{\text {core }_{\mathfrak{L}(G)}} M=$ core $_{G} M$ (the usual core of $M$ in $G$ in the group-theoretical sense).

Lemma 4.3 (see [3], Lemma 3). Let $G$ be a group, and let $M$ be a maximal subgroup of finite index of $G$. If $M$ is not modular in $G$, then the largest modular subgroup of $G$ contained in $M$ is normal in $G$.

Theorem 4.4. Let $G$ be an infinite group, and let $H$ be a proper subgroup of finite index of $G$. Then the number of prime factors of $|G: H|$ (with multiplicity) is the $\operatorname{sum} \sum_{i=0}^{t-1}\left\|H_{i+1}: H_{i}\right\|$, where

$$
H=H_{0}<H_{1}<\ldots<H_{t}=G
$$

is a finite chain of subgroups such that $H_{i}$ is an $f$-maximal subgroup of $H_{i+1}$ for each $i=0,1, \ldots, t-1$.

Proof. Assume first that $H_{i}$ is a modular subgroup of $H_{i+1}$ for some non-negative integer $i<t$, so that $\left\|H_{i+1}: H_{i}\right\|=1$ as we already observed; on the other hand, it is well known that in this case the index $\left|H_{i+1}: H_{i}\right|$ is a prime number (see [4, Lemma 5.1.2). Suppose now that $H_{i}$ is not modular in $H_{i+1}$, and let $K_{i}$ be the normal core of $H_{i}$ in $H_{i+1}$. By Lemma 4.3, $K_{i}$ is the largest modular subgroup of $H_{i+1}$ contained in $H_{i}$, and hence we have

$$
\begin{gathered}
\left\|H_{i+1}: H_{i}\right\|=\left\|\left[H_{i+1} / K_{i}\right]\right\|-\left\|\left[H_{i} / K_{i}\right]\right\| \\
\quad=\left\|\mathfrak{L}\left(H_{i+1} / K_{i}\right)\right\|-\left\|\mathfrak{L}\left(H_{i} / K_{i}\right)\right\| .
\end{gathered}
$$

Since $H_{i+1} / K_{i}$ is a finite group, it follows from Corollary 3.5 that the lattice index $\left\|H_{i+1}: H_{i}\right\|$ is the number of prime factors of $\left|H_{i+1} / K_{i}: H_{i} / K_{i}\right|=\left|H_{i+1}: H_{i}\right|$. The theorem is proved.

Corollary 4.5. Let $\varphi$ be a projectivity between the groups $G$ and $\bar{G}$, and let $H$ be a subgroup of finite index of $G$. Then the indices $|G: H|$ and $\left|\bar{G}: H^{\varphi}\right|$ have the same number of prime factors. 


\section{REFERENCES}

[1] E. Previato: "Gruppi in cui la relazione di Dedekind è transitiva", Rend. Sem. Mat. Univ. Padova 54 (1975), 215-231. MR.0466319 (57:6199)

[2] D.J.S. Robinson: "A Course in the Theory of Groups", Springer, Berlin (1992). MR1261639 (94m:20001)

[3] R. Schmidt: "Verbandstheoretische Charakterisierungen der Endlichkeit des Indexes einer Untergruppe in einer Gruppe", Arch. Math. (Basel) 42 (1984), 492-495. MR0756887 (86g:20035)

[4] R. Schmidt: "Subgroup Lattices of Groups", de Gruyter, Berlin (1994). MR1292462 (95m:20028)

[5] G. Zacher: "Una caratterizzazione reticolare della finitezza dell'indice di un sottogruppo in un gruppo", Atti Accad. Naz. Lincei Rend. Cl. Sci. Fis. Mat. Natur. (8) 69 (1980), 317-323. MR0690298 (84f:20027)

Dipartimento di Matematica e Applichzioni, Università di Napoli "Federico II", Complesso Universitario Monte S. Angelo, Via Cintia, I - 80126 Napoli, Italy

E-mail address: mdefalco@unina.it

Dipartimento di Matematica e Applicazioni, Università di Napoli "Federico II", Complesso Universitario Monte S. Angelo, Via Cintia, I - 80126 Napoli, Italy

E-mail address: degiovan@unina.it

Dipartimento di Matematica e Applicazioni, Università di Napoli "Federico II", Complesso Universitario Monte S. Angelo, Via Cintia, I - 80126 Napoli, Italy

E-mail address: cmusella@unina.it

Mathematisches Seminar, Universität Kiel, Ludwig-Meyn Strasse 4, D - 24098 Kiel, GERMANY

E-mail address: schmidt@math.uni-kiel.de 